Revista de Investigación Educativa 25

julio-diciembre, 2017 | ISSN 1870-5308 | Xalapa, Veracruz

Instituto de Investigaciones en Educación | Universidad Veracruzana

Etnizando metodologías de investigación social: formación para el fortalecimiento del liderazgo de mujeres indígenas

Indigenizing Social Research Methodologies: Training Experience for the Strengthening of Women's Leadership

Dra. Dolores Figueroa Romero

Catedrática

Centro de Investigación y Estudios Superiores en

Antropología Social-Ciudad de México, México

figueroa.lola@gmail.com

Dra. Araceli Burguete Cal y Mayor

Catedrática

Centro de Investigación y Estudios Superiores en

Antropología Social-Sureste, México

araceli burguete@yahoo.com.mx 
A partir de la descripción de enfoques y procesos de enseñanza de investigación del Diplomado para el Fortalecimiento del Liderazgo de las Mujeres Indígenas, coordinado por la Universidad Indígena Intercultural del Fondo Indígena y el Centro de Investigaciones y Estudios Superiores en Antropología Social, se desarrollará una reflexión sobre "la etnización” de la investigación social y la producción de conocimiento cultural y políticamente pertinente para el movimiento de mujeres indígenas organizadas en América Latina. En específico, nuestro análisis se centrará en mostrar los escenarios de disputa donde facilitadoras y lideresas se enfrentaron ante el reto de desmontar la colonialidad de la construcción del conocimiento en las dinámicas de enseñanza y procesos de adecuación de métodos de investigación. Las particulares experiencias de conducción del trabajo de campo de las alumnas mostrarán su creatividad al adaptar y adoptar metodologías que les permitieron visibilizar el aporte político de las mujeres indígenas al desarrollo del activismo indígena local.

Palabras clave: Diplomados; mujeres; educación intercultural; formación de sujetos sociales; liderazgo; producción del conocimiento.

Based on an ethnographic description of the approaches, learning processes and final research products of the Diploma for the Strengthening of Women's Leadership coordinated by the Indigenous Fund's Intercultural Indigenous University and the Center for Research and Advanced Studies in Social Anthropology (UII-CIESAS), this essay reflects upon the indigenization of social research and knowledge production designed to meet the cultural and political needs of the indigenous women's movement in Latin America. Specifically our analysis will focus on showing scenarios of dispute where facilitators and leaders faced the challenge of dismantling the coloniality of the knowledge construction in teaching dynamics and processes of adequacy of research methods. Finally, the students' own fieldwork experiences show their creativity in adapting and adopting methodologies that allowed them to make visible the political contribution of indigenous women to the local indigenous activism.

Keywords: Diploma; women; intercultural education; leadership; social subjects formation; knowledge production. 
Etnizando metodologías de investigación social: formación para el fortalecimiento del liderazgo de mujeres indígenas

Dolores Figueroa Romero y Araceli Burguete Cal y Mayor

\title{
Etnizando metodologías de investigación social: formación para el fortalecimiento del liderazgo de mujeres indígenas
}

\author{
Indigenizing Social Research Methodologies: Training \\ Experience for the Strengthening of Women's Leadership
}

\section{Introducción}

- A qué hacemos referencia con "la etnización" de la investigación social y sus ¿métodos? Diríamos que se trata de un proceso que implica una crítica y resistencia a la hegemonía del conocimiento occidental, lo que conlleva — entre otras cosas - a cuestionar de manera sistemática los supuestos dominantes universalistas de las ciencias sociales y adaptar metodologías convencionales de investigación, de tal manera que incluyan perspectivas y métodos que tienen su origen o están relacionados con conocimientos indígenas, lenguajes, mundos de vida, experiencias, filosofías y metáforas, o que transforman y re-significan aquellas que se producen en la academia formal (Walsh, Mignolo \& García, 2006). Se trata de un proceso que está influido e inspirado en reclamos políticos de reivindicación étnica, en posicionamientos descoloniales, y perspectivas subjetivistas y situadas de la teoría feminista (Bertely, 2013; Chilisa, 2002; Medina \& Baronnet, 2013; Smith, 1999; Walsh, 2009).

El proceso de la "etnización de la investigación" reta tanto a investigadores consolidados como aprendices de las ciencias sociales a invocar el conocimiento indígena para conformar o encontrar nuevos paradigmas teóricos, nuevas herramientas de análisis, métodos para el levantamiento de datos y la inclusión de fuentes de conocimiento orales que están socialmente invisibilizadas (Cunningham, 2002; Guidugli \& Cunningham, 2009). El énfasis es que los investigadores - tanto indígenas como no indígenas - inmersos en un diálogo intercultural puedan combinar teoría, enfoques teóricos y metodologías occidentales con cosmovisiones y tradiciones de pensamiento indígena. Este proceso requiere de una doble sensibilidad, investigadoras implicadas en darle un espacio a los procesos auto-reflexivos indígenas y la participación de aprendices comunitarios e investigadoras indígenas en la producción de co- 
nocimiento desde sus necesidades políticas y esfuerzos inquisitivos (Dietz \& Mateos, 2015). Es entonces cuando la etnización de métodos de investigación convencionales puede verse como resultado del ejercicio teórico-político de los actores involucrados, así como de la fusión de enfoques de varias disciplinas en la generación de nuevos conceptos, enfoques y conocimientos.

Este ensayo aborda aspectos problemáticos de la colaboración entre la academia y las organizaciones indígenas en el desafío de cuestionar la hegemonía de las metodologías de investigación social convencionales y potencializar nuevas maneras de abordar temas como etnicidad, género, conocimiento y poder desde una perspectiva de-colonial e inter-seccional (Lugones, 2015; Rappaport, 2015). En particular, agregaríamos que la experiencia aquí descrita da cuenta de un ejercicio de contestación que irrumpió desde el mismo protagonismo de las mujeres indígenas organizadas que decidieron impulsar un proceso de formación de liderazgos asertivos en colaboración con académicas y activistas de derechos humanos (Cunningham, Mendizábal \& Martínez, 2015; Figueroa, 2010; Vargas, López \& Burguete, 2012).

La experiencia formativa del "Diplomado para el Fortalecimiento del Liderazgo de las Mujeres Indígenas" la coordinó académicamente el Centro de Investigaciones y Estudios Superiores en Antropología Social (CIESAS) ${ }^{1}$ y fue ofertada por el Fondo Indígena a través de la Universidad Indígena Intercultural (UII) ${ }^{2}$ a lideresas indígenas -jóvenes y experimentadas - interesadas en el fortalecimiento de su liderazgo, en desarrollar capacidades de investigación y en adquirir conocimiento especializado político-legal para elevar su desempeño en procesos de gestión, incidencia y desarrollo comunitario. Es importante ubicar el diplomado como producto del trabajo estratégico de varias lideresas indígenas latinoamericanas, que desde hace varios años actúan de manera coordinada a fin de fortalecer y consolidar las redes transnacionales de mujeres indígenas que hacen trabajo de incidencia a nivel local, regional y global

1. La coordinación académica del Diplomado fue realizada por la Dra. Araceli Burguete Cal en sus cuatro emisiones.

2. La UII es una iniciativa educativa regional impulsada por el Fondo Indígena que acciona como una red en colaboración con Centros Académicos Asociados. Su objetivo es la formación de profesionales indígenas con capacidad de liderazgo para que puedan asumir, desde una perspectiva intercultural, tareas de articulación e incidencia en procesos económicos, políticos y sociales que determinan la vida de los pueblos indígenas en América Latina. La Ui tiene una amplia oferta de posgrados y diplomados sobre temas como salud intercultural, derechos indígenas, gestión pública y gobernabilidad, desarrollo con identidad, políticas públicas y educación intercultural bilingüe. http://www. fondoindigena.org/drupal/es/formacion 
Dolores Figueroa Romero y Araceli Burguete Cal y Mayor

(Burguete, 2013; Figueroa, 2010). La formación de estas redes se relaciona fundamentalmente con el incremento de las capacidades de lideresas situadas a distintos niveles organizativos y políticos, que coadyuvan a dinamizar la circulación de información, vital para el quehacer político global y local del movimiento indígena contemporáneo (Martí i Puig, 2004; Sikkink, 1993; Valladares, 2008).

El Diplomado tuvo su primera edición en 2010, repitiéndose en cuatro ocasiones más, en los años 2011, 2012 y 2013. El total de alumnas sumando las cuatro generaciones fue de 98, de las cuales se graduaron 87 , logrando con ello un promedio de 88.7 de eficiencia terminal (Burguete, 2013). El diplomado estuvo organizado en cuatro momentos pedagógicos: a) una primera concentración presencial; b) una fase virtual intermedia; c) una sesión presencial conclusiva, y d) presentación de resultados de investigación. La convocatoria abierta de ingreso fue dirigida a mujeres con los siguientes perfiles: lideresas de base comunitaria y trayectoria organizativa; profesionistas indígenas ligadas a procesos organizativos, educativos, políticos o de desarrollo; profesionistas indígenas con trayectoria de trabajo con organizaciones indígenas; lideresas indígenas y/o mujeres indígenas electas en cargos de elección, y lideresas indígenas activistas de derechos humanos. Un importante requisito de aceptación fue que las estudiantes contaran con una tentativa de propuesta de investigación y el aval de su organización y/o autoridad indígena. Los resultados de las investigaciones se materializaron en reportes, diagnósticos e informes de variada naturaleza temática. ${ }^{3}$

3. Se elaboraron 87 proyectos de investigación que fueron desarrollados entre 2010 y 2013. Más allá de las particularidades que cada generación del diplomado tuvo — variantes en cuanto a la participación de docentes, el énfasis formativo y la diversidad de las alumnas-, es posible trazar algunas generalidades sobre la riqueza temática que las alumnas produjeron. De manera general se podría decir que los proyectos de investigación respondieron a seis grandes bloques temáticos:

- Temas relativos a procesos de formación de liderazgo de las mujeres indígenas en distintos espacios como organizaciones mixtas, de mujeres, de jóvenes y organizaciones comunitarias.

- Diagnósticos exploratorios sobre problemática de la situación de las mujeres indígenas -salud reproductiva, violencia doméstica, pobreza - y la vulnerabilidad para lograr el reconocimiento de sus derechos tanto a nivel local como nacional.

- Reflexiones sobre la centralidad de la recuperación de saberes indígenas (medicina tradicional, lengua, identidad y cosmovisión) para el fortalecimiento de la identidad.

- Análisis sobre la posición de la mujer en procesos de impartición de justicia desde el derecho indígena y los conflictos inter-legales con el derecho positivo.

- Reflexiones y análisis sobre la relación entre la educación, la lucha indígena y la profesionalización del liderazgo indígena.

- Estudios sobre el impacto del cambio climático en la problemática de seguridad alimentaria y procesos productivos.

- Desplazamiento forzado, migración, urbanización y pérdida de la identidad inter-generacional. 
El programa curricular estuvo organizado en cuatro áreas de concentración temática que guiaron la práctica docente de los facilitadores de manera interconectada:

I. La primera refiere a los enfoques conceptuales en los que descansó el diplomado, como el enfoque de derechos, el del género y la interculturalidad.

II. La segunda área concentró enfoques epistemológicos y perspectivas críticas a las formas de producción y validación del conocimiento convencional occidental, tratando a su vez de abrir el aprendizaje a formas de conocimiento (oral e indígena) que no han sido valoradas o incluidas en los espacios de formación universitaria.

III. La tercer área temática concentró herramientas metodológicas y ejercicios reflexivos tendientes a fusionar métodos participativos de investigación con recursos propios y saberes indígenas re-significados. Por ejemplo la llamada "metodología de la re-conexión" buscó favorecer diálogos y facilitar el aprendizaje horizontal entre las participantes por encima de las diferencias, como las características culturales de sus pueblos, los contextos rural-urbano, las diferencias de género (hombre-mujer), las distancias generacionales (hijas-madres-abuelas), diversidad de religiones y posicionamientos político-ideológicos. Por último,

IV. El área temática formativa incluyó temas como la autodeterminación indígena, la espiritualidad, la complementariedad-dualidad, los roles socio-políticos de las mujeres indígenas, y la historia del movimiento indígena en América Latina.

El aporte del Diplomado a la producción de conocimiento por mujeres indígenas puede analizarse a través de los siguientes lentes. Para propósitos de este ensayo priorizaremos: 1) el lente de la experiencia de las facilitadoras indígenas y no indígenas que, inspiradas por la crítica tanto de las ciencias sociales como del feminismo, buscaron experimentar y adecuar los métodos de enseñanza y el contenido curricular de los módulos; 2) el lente de la experiencia de investigación de las alumnas que incluyó el trabajo de campo y la politización de su identidad étnica y de género, como investigadoras indígenas, y 3 ) una reflexión desde el propósito político de lo investigado por las alumnas, pues se buscó que los temas coadyuvaran a la comprensión de sus condiciones como mujeres y de los lugares que les son socialmente asignados.

El cuerpo del ensayo está organizado primero con una sección teórica donde brindamos los elementos analíticos de la razón descolonizadora de la producción del conocimiento, los acercamientos a este compromiso desde la antropología y el feminismo decolonial, y el aporte sustancial que ha brindado el movimiento organizado de mujeres indígenas. De seguido incluimos una sección sobre la experiencia de enseñanza de investigación en sí y los escenarios de disputa sobre la construcción de la 
otredad y el dato etnográfico, donde alumnas y facilitadoras nos vimos tensionadas por la identidad del sujeto que investigan en una suerte de estar dentro y fuera de la comunidad (Lugones, 2015; Rappaport, 2015). Continuamos con una reflexión sobre el trabajo de campo de dos alumnas del diplomado que discurren sobre la identidad emergente de asumirse como "investigadoras", el ejercicio de hacer suyas las herramientas y la aplicación de formas de trabajo acorde a las exigencias de su comunidad. Por último, cerraremos con una consideración sobre los resultados positivos de la etnización de la investigación social por las actoras políticas posicionadas.

\section{La investigación social, la descolonización y el activismo contemporáneo de las mujeres indígenas}

Ya varios antropólogos y académicos de las ciencias sociales en América Latina han abordado el tema de la complejidad ética, política y teórica de la relación entre cientistas sociales no indígenas y líderes indígenas en la producción de conocimiento para la liberación de los pueblos (Hale, 2008; Jimeno, Murillo \& Martínez, 2012; Lugones, 2015; Rappaport, 2015; Varese, Delgado \& Meyer, 2008). En estos debates sobre el poder y las jerarquías de conocimiento en los procesos de transformación de las relaciones del Estado y los pueblos indígenas, los antropólogos han sido inspirados por una ética de izquierda por la liberación que, aunque sea bien intencionada, ha sido atinadamente interpelada por el activismo indígena organizado en América Latina (Hale, 2008; Varese et al., 2008). Gracias a la crítica de la militancia indígena organizada y a los beneficios de la deconstrucción post-estructural y el post-colonialismo, el acompañamiento de la antropología a la liberación de los pueblos indígenas ha sido cuestionado desde varios ángulos. En el campo del pensamiento político subversivo, Varese et al. (2008) señalan que los líderes indígenas, en su periplo de tránsito por movimientos de izquierda, tomaron consciencia de la arrogancia e insensibilidad de los marxista latinoamericanos hacia la especificidad de las demandas culturales indígenas y las aspiraciones de autodeterminación y autonomía (p. 381). En el campo de la producción del conocimiento sobre las relaciones étnico-nacionales, fue preciso develar la colonialidad de la antropología que le otorga a sus expertos el poderse construir la categoría de "otredad", cuando por otro lado, los líderes indígenas cuestionan de maneras poderosas construcciones estáticas de la indigenidad, interpelando a la autoría antropológica para certificar lo que es indígena y no indígena desde una mirada externa. 
Dolores Figueroa Romero y Araceli Burguete Cal y Mayor

A la luz de este campo minado de tensiones con aliados y externos que fue la regla en los años sesenta y setenta, ahora, en la era del neoliberalismo multicultural, las organizaciones indígenas contemporáneas han buscado explorar nuevos marcos de colaboración que les permitan escalar sus capacidades y foco de acción política de la micro-política comunitaria (auto-referida y contenida) a la macro-política indígena, donde toman lugar acciones coordinadas en varios niveles geo-políticos (Brysk, 2000; Niezen, 2009; Pallares, 2007). Este escalamiento y sofisticación de las estrategias de trabajo activista indígena ha aterrizado en varios escenarios como el educativo-formativo, el jurídico-territorial y el político-electoral. Específicamente en el área de educación informal, educación superior y formación de liderazgos, las dinámicas de colaboración entre indígenas y no indígenas han tenido una particularidad creativa, pero al mismo tiempo compleja (Cunningham et al., 2015; Dietz \& Martí i Puig, 2013; Dietz \& Mateos, 2015; Mato, 2013; Vargas, López \& Burguete, 2012).

Como autoras de este ensayo y participantes en la realización del Diplomado 4 en esfuerzos colegiados de equipos de enseñanza interdisciplinarios, queremos trenzar nuestro análisis en relación con dos elementos clave de crítica. Uno hace eco del camino reflexivo sobre el poder de la autoría antropológica y, agregaríamos, feminista para representar a la "otra" - la mujer indígena- en la producción del conocimiento sobre la condición femenina indígena (Cervone et al., 1998; Marchand \& Parpart, 1999). Segundo, el impacto del pensamiento estratégico del movimiento organizado de mujeres indígenas, que desde hace dos décadas ha ido tomado un rol central en la creación de condiciones para visibilizar sus demandas como actoras políticas, con una especificidad particular en el universo de movimientos subalternos (Figueroa, 2006; Valladares, 2008). En concreto, estas consciencias representan, a nuestro entender, dos polos clave dentro de un campo minado de compromisos políticos y racionalidades que determinaron de manera diferenciada el actuar de todos los involucrados en esta experiencia formativa (Burguete, 2013).

En tanto antropólogas comprometidas con el movimiento de mujeres indígenas, hemos sido tocadas por la necesidad de ser reflexivas sobre la racionalidad feminista, en generalizar las opresiones de las mujeres en el mundo indígena y los métodos para dinamizar procesos de cambio de los regímenes de género. Para ello hemos incorporado perspectivas como la inter-seccionalidad, que nos ha ayudado a dimensionar la

4. La coordinación académica del Diplomado estuvo a cargo de la Dra. Araceli Burguete Cal y Mayor, mientras que la docencia en línea de los módulos de liderazgo y género estuvieron a cargo de la Dra. Dolores Figueroa Romero. Ambas autoras de este ensayo. 
Dolores Figueroa Romero y Araceli Burguete Cal y Mayor

co-construcción de categorías como sexo, raza, etnicidad y clase en la constitución de las identidades y escenarios de lucha de las mujeres indígenas (Cumes, 2009; Lugones, 2015). Esta mirada compleja de la construcción social de las mujeres indígenas como actoras sociales cuestiona las relaciones de poder entre lideresas y aliadas no indígenas, de-centralizando de manera poderosa las categorías, racionalidades y órdenes explicativos. Para complementar, hemos sido testigos también desde mediados de los años noventa de diversos foros continentales y encuentros organizados por mujeres indígenas, desde donde ellas impulsan procesos organizativos que les permiten posicionarse de manera más ventajosa en relación con sus contrapartes varones indígenas y donantes (Dixon \& Sánchez, 2003). Dentro de estas acciones estratégicas, la educación y la formación han sido uno de los mecanismos vitales que las mujeres indígenas identifican para facilitar la obtención de herramientas, conocimiento y capital social para fortalecerse políticamente como sujetos de derecho en contra de la opresión estructural y social que las discrimina de manera sistemática (Cunningham, 2002; Dixon \& Sánchez, 2003; Figueroa, 2006, 2010).

Como educadoras y cercanas a procesos políticos de base conocemos de primera mano la evidencia de que las mujeres indígenas efectivamente tienen un acceso restringido a la educación formal e informal. Encontramos problemáticas las intervenciones en el ámbito comunitario de muchos Organismos No Gubernamentales de desarrollo y conservación ambiental que, aunque sean bien intencionadas, con frecuencia tratan a las beneficiarias como simples receptoras de información y conceptos diseñados desde afuera. Creemos importante mostrar nuestro distanciamiento del concepto de capacitación empleado en experiencias formativas informales y programas de empoderamiento que focalizan su trabajo con mujeres indígenas a fin de intervenir sobre los ordenes de género en la comunidades, sin ser reflexivos sobre contextos culturales concretos y las formas locales en como las mujeres visualizan el goce de una vida libre de violencia y con dignidad (Cervone, 2002; Segato, 2014).

A la luz de lo anterior es que reconocemos que, en el campo de la formación, la agencia de las mujeres indígenas organizadas y los profesionistas indígenas y/o lideresas políticas es central para dar sentido a la disputa de enfoques y contenidos. Siguiendo la secuela de la reunión de Barbados, diríamos que los profesionistas indígenas y líderes reclaman su protagonismo al frente de todos los procesos educativos tendientes a fortalecer y empoderar a sus pueblos (Dietz \& Martí i Puig, 2013; Vargas et al., 2012). En específico añadiríamos que el Diplomado del que aquí se da cuenta fue, en gran medida, resultado de una larga cadena de negociaciones y alianzas construidas por 
mujeres indígenas organizadas a diferentes escalas geo-políticas — tanto en espacios de género mixtos como de sólo mujeres - con actores institucionales, a fin de crear condiciones para su realización.

La oferta final de contenidos curriculares para las alumnas del Diplomado fue resultado de la sinergia de tres elementos: primero, un diagnóstico exploratorio sobre demandas específicas de formación para mujeres indígenas que se realizó con dirigentas y secretarias de asuntos de la mujer en diversas organizaciones mixtas en América Latina; segundo, la labor de síntesis y formulación que hiciera la coordinadora académica del CIESAs de cara a lograr una propuesta curricular promisoria y realizable, y tercero, la labor de incidencia de lideresas que en el seno del Equipo Gestor del Fondo Indígena ${ }^{5}$ tuvieron a bien concretizar propuestas para lograr que las perspectivas $y$ epistemologías de los pueblos indígenas en la producción de conocimiento se vieran reflejadas siguiendo lineamientos clave.

La estrategia logística y pedagógica incluyó la selección de un equipo especializado de expertos (sabios indígenas de la "Catedra Indígena Itinerante" y antropólogos) para la docencia presencial y en línea. La gestión de fondos para asegurar los medios técnicos y recursos monetarios que reunieran a un grupo de alumnas representativo de lideresas indígenas de toda América latina. La formulación de un plan de enseñanza de elementos estratégicos para romper con la dependencia del quehacer profesional de antropólogos para la generación de conocimiento sobre mujeres indígenas que resultara políticamente relevante para ellas, en tanto protagonistas de la lucha organizada de sus pueblos. Para lograrlo, por ejemplo, se necesitó idear mecanismos que visibilizaran la mirada femenina indígena sobre procesos colectivos de lucha, que aunque ya hubieran sido narrados con anterioridad carecían de la inclusión del aporte de las mujeres en la producción cultural, biológica y política de sus pueblos. Esta tarea, por demás compleja, requirió de-construir narrativas ciegas al género sobre la emergencia del movimiento indígena en los noventa, traer a la luz historiografías de movilizaciones populares desde el punto de vista de las mujeres y/o lideresas históricas, e incentivar procesos auto-reflexivos entre las alumnas que las hicieran explorar las vetas de rebelión y protagonismo femenino en luchas populares.

5. La experiencia y liderazgo de la doctora Mirna Cunningham fue central en todo el proceso; además que se contó con el apoyo de la cooperación alemana (GIZ) para el financiamiento del diplomado. 
Dolores Figueroa Romero y Araceli Burguete Cal y Mayor

\section{Re-significando métodos de investigación y desmantelando la supremacía de la antropología occidental en la representación de la otredad}

En esta sección describimos y analizamos las dinámicas que se dieron en el adiestramiento de las alumnas sobre la adquisición de sensibilidades y métodos para la investigación social. Este contenido jugó un papel central en potencializar en las alumnas capacidades para realizar procesos inquisitivos y de levantamiento de datos que les permitieran desarrollar sus investigaciones. Para poder llevar esto a buen fin se requirió del actuar coordinado y colaborativo de varios docentes en el diplomado, quienes desde distintos ámbitos y roles facilitaron la adquisición de elementos para aprender a ser investigadoras.

Estas dinámicas se llevaron a cabo en las sesiones presenciales al inicio del Diplomado, donde se introdujo información sobre métodos y metodologías para la investigación social desde una perspectiva dialógica, y con énfasis en la etnización de la investigación. Para realizar este proceso, se solicitaba la participación de facilitadores indígenas académicos, quienes incentivaban reflexiones sobre la teoría des-colonial y una crítica a la producción de conocimiento hegemónico. El objetivo de esta área temática fue darle centralidad a las epistemologías indígenas y a la importancia de estudiar prácticas sociales indígenas de manera apropiada metodológica y éticamente. A fin de avanzar en este aspecto, se buscó visibilizar el valor epistémico de varios espacios, momentos y dinámicas de producción de conocimientos, como las enseñanzas ancestrales de los abuelos; las dinámicas de participación por género y edad en las asambleas comunitarias; las historias de vida de mujeres en las comunidades y su participación en la economía local; la pedagogía del trabajo colectivo de las mingas; los rituales de la vida familiar, de la chacra, de la muerte; los espacios tradicionales de transferencia generacional de conocimiento, y demás. La intención era mostrar el potencial epistémico de estos espacios cotidianos y motivar a las estudiantes a realizar ejercicios de observación participante para recuperar los métodos de transmisión de los saberes ancestrales y sistematizar la información generada.

Entre las dificultades en dicha etapa estaba despertar el interés investigativo de las participantes en el universo cognitivo indígena, mostrar la importancia de aprender métodos para el registro de la información y otorgar el rango de conocimiento a los saberes propios. Esta tarea implicó asistir a las participantes en el desarrollo de sus capacidades, para convertir "la observación" en datos y para "registrar" conversa- 
ciones en la cotidianeidad. Para completar este desafío, las facilitadoras socializaban información sobre las herramientas para llevar a cabo la investigación convencional, tales como las conversaciones familiares informales (la "metodología del fogón" le llamó una estudiante colombiana), la historia oral, la observación participante, los talleres de trabajo y los grupos focales. Todos estos métodos investigativos se les presentaron como potencialmente útiles para recaudar la información requerida para sus proyectos de investigación, no sin antes recordarles que las técnicas aprendidas debían ser adaptadas a las circunstancias particulares de cada tema de estudio, requiriendo principalmente conversaciones y diálogos con los actores involucrados en la temática de investigación. También hubo orientación para que las alumnas buscaran asesoría de sabios locales que las guiaran para reconocer distintas formas de pensamiento indígena, registrarlo y contextualizarlo, potenciando así el diálogo intra-cultural, inter-generacional, intra e inter géneros.

Las multi-dinámicas de enseñanza siempre estuvieron fuertemente determinadas por lo que podríamos nombrar como "la paradoja de la antropología y la paradoja de los indígenas que estudian antropología o que hacen antropología” (Varese et al., 2008, p. 378). Esta paradoja reside en dos cosas fundamentales: 1) el hecho de aprender métodos $\mathrm{y}$ formas de racionalizar desde una disciplina moderna fundada en paradigmas racionalistas, propios del iluminismo europeo, que privilegian extracción y sistematización de datos para avanzar interpretaciones sobre culturas no occidentales; 2) el adiestramiento del antropólogo que construye su vivencia liminal y cognitiva al estar afuera de un colectivo culturalmente diferenciado y que se tiene como desconocido. Ese sentido de conocer y construir conocimiento desde la posicionalidad externa que recrea construcciones exotizadas de la otredad que terminan siendo legitimadas. Pero en nuestro caso, o en el de los antropólogos embarcados en dinámicas colaborativas de investigación, ¿qué pasa cuando el que está inmerso en procesos de aprendizaje y entrenamiento es indígena, o son una amplia gama de sujetos diversos que se ubican de manera fluida en un espectro complejo de subjetividades auto-adscritas como indígenas?

Para responder a esta pregunta quisiéramos hacer referencia a experiencias colaborativas que se han dado en América Latina, donde se han explorado distintos caminos para descentralizar la autoría del antropólogo en representar a otros y facilitar la adquisición de destrezas para que esos otros puedan también representarse a sí mismos. De un amplio espectro de experiencias de colaboración y co-teorización retomaremos el trabajo de Rappaport (2015), quien se ha ocupado en mostrar los resultados promisorios de procesos de investigación de largo aliento con organizacio- 
nes indígenas en temas educativos; también se le reconoce por su capacidad de hacer visible y reflexionar sobre la complejidad del diálogo de saberes entre diversos actores y espacios liminales. Rappaport hace eco en su reflexión de los contextos políticos comunes donde tanto antropólogos como líderes indígenas comparten la preocupación por la construcción de naciones justas, y por la elaboración conjunta de categorías teóricas nuevas en el proceso de recolección de datos en experiencias concretas de investigación etnográfica. Rappaport (2015) señala de manera acuciosa que este ejercicio resulta creativo cuando el control de la elaboración etnográfica pasa al sujeto interno, es decir, a un actor o actores políticos que están situados dentro de la comunidad indígena, asumiendo también la existencia de una contraparte externa que cede y se mantiene observante de los procesos políticos de la comunidad (p. 331). La distinción y oposición conceptual adentro y afuera (del mundo indígena) es fructífera metodológicamente porque permite ver una serie de diálogos ubicados en un espectro fluido de intercambios entre un grupo heterogéneo de externos (asesores, colaboradores) y un grupo diverso de actores internos también. La noción de adentro no se refiere únicamente a la comunidad originaria y tradicional, sino también a la frontera de espacios (geográficos e institucionales) donde líderes indígenas se movilizan a fin de hacer trabajo de incidencia a favor de sus pueblos (p. 337).

Para nuestro caso particular de formación, el énfasis en la diversidad de formas de "estar adentro" y en actores internos resulta muy útil para nombrar y conceptualizar los variados posicionamientos de las alumnas del diplomado, entendiéndolas como un grupo heterogéneo de mujeres indígenas, profesionistas y lideresas de base, que en su mayoría han experimentado varios grados de lejanía y enajenación de su propia cultura y comunidad. La heterogeneidad de las estudiantes (en términos de edad, formación profesional y residencia) da cuenta de las muy variadas maneras de estar adentro o buscar estar adentro mediante procesos de reconexión y reconocimiento en las epistemologías del mundo indígena. Otro elemento importante del aporte de Rappaport es el hecho de que la versatilidad de la distinción adentro y afuera, descansa sobre entendimientos constructivistas de la cultura indígena, permitiendo y ofreciendo a las alumnas del Diplomando flexibles maneras de articular su pertenencia indígena a partir de un trabajo auto-reflexivo y político. Nosotras como facilitadoras y también las estudiantes, lo indígena no lo definimos restringido a formas de vida circunscritas a una comunidad cerrada y rural, sino en nuevas formas de ser y recrear indigenidad con un poderoso sentido universal de cuidado, de encuentro y de conexión con el mundo natural y espiritual de muy variadas formas. 
Aquí quisiéramos regresar al punto conflictivo del aprendizaje de métodos de investigación social, que fue la reflexión con la que iniciamos esta sección, con el propósito de preguntarnos sobre los procesos de aprendizaje de un espectro complejo de individuos auto-adscritos como indígenas de maneras muy variadas. Primero, porque queremos resaltar el hecho de la incomodidad que en ellas despertó el tema del aprendizaje de métodos de levantamiento de datos tradicionalmente usados por la antropología, y segundo, por el carácter interno y desde adentro de su subjetividad como lideresas que negocian agendas en varios espacios políticos. Ahí sí la dicotómica diferencia entre adentro y fuera se agudizó por el hecho del aprendizaje de métodos científicos de ciencias sociales considerados externos al mundo indígena y por la recepción negativa que tendrían en la comunidad al hacer uso de esos instrumentos con su propia gente.

En el momento presencial de la enseñanza de metodologías cualitativas de recolección de datos en campo nos dimos cuenta de un des-encuentro con las estudiantes, sobre todo de cara a sus dilemas éticos de convertirse en investigadoras, y la perspectiva de ver a su propia comunidad como un "objeto de estudio". En nuestro entender interpretamos esa incomodidad ligada a significados negativos de "la investigación y prácticas investigativas”, que la investigadora indígena maorí Smith (1999) señala como "la palabra más sucia en el vocabulario indígena" (p. 1). Preocupada por el despojo cognitivo en contextos indígenas, Smith (1999) advierte en contra de iniciativas que no son sensibles a los intereses de los pueblos indígenas y que ultimadamente responden a intereses neo-coloniales de dominación (p. 16). En el caso de nuestras estudiantes, la mayoría de ellas había tenido experiencias o sabido de visitantes externos que extrajeron importante información de sus comunidades —inclusive hubo narrativas de casos de individuos que tomaron títulos de posesión territorial de la colonia- y que nunca retribuyeron nada a cambio. A la luz de esas experiencias las alumnas reiteraron de manera enfática su compromiso por desarrollar procesos de investigación respetuosos desde una perspectiva propia, aunque sin una idea práctica de cómo hacerlo.

A fin de ayudar a las estudiantes a superar las múltiples fuentes de inconformidad, como profesoras y facilitadoras tratamos de ubicar sus intereses y experiencias al centro de las dinámicas de clase. Impulsamos la selección de temas de investigación que fueran centrales y vitales para las comunidades de las alumnas, como: despojo territorial, contaminación ambiental, violencia de género, mortalidad materno-infantil, acceso a justicia y formas de desarrollo propias, entre otras. De igual manera, con 
quienes estuvimos agradecidas de que entre las estudiantes hubiera lideresas más experimentadas con quienes, en diálogo con nosotras, facilitamos el aprendizaje de las más jóvenes sobre la aplicación de métodos de recolección de datos para diferentes propósitos, como entrevistas a ancianos, observación de ritos de curación, participación en la preparación de comida, rezos, fiestas, grabación de cantos, historias y leyendas, aprendizaje de artes de tejido o cerámica. Un punto de tensión adicional fue que en varios sentidos nuestros mensajes - como facilitadoras no-indígenas y feministas - fueron tomados como imposición de epistemologías occidentales y, ciertamente, para mitigar sentimientos de sospecha por parte de las alumnas, necesitamos de la gentil intervención de lideresas experimentadas.

La explicación desde la experiencia personal de liderazgos indígenas haciendo investigación facilitó entre las jóvenes una segunda mirada más positiva sobre el uso de los métodos de investigación social. Específicamente, sobre el tema de la sistematización de los datos de campo, la mediación de los sabios indígenas con las alumnas fue atinada en el siguiente sentido. Era vital que las alumnas tuvieran cuidado en utilizar herramientas del registro de información en campo. Esta enseñanza implicó que las alumnas problematizaran los usos adecuados de la grabadora y la libreta de campo. Muchas de las estudiantes se reconocían como activas participantes de los quehaceres colectivos de sus pueblos y conocedoras "del adentro" de sus culturas, de ahí que en ciertos, los momentos más álgidos de la discusión, ellas valoraran el uso de la grabadora como un insulto y ofensa para sus mayores por pretender aplicar en ellos formas de registro no pertinentes a su marco cultural. Ante esta serie de observaciones insistentes y acuciosas, un participante de amplio conocimiento en métodos antropológicos explicó la necesidad de ser sistemático en registrar dinámicas sociales - aunque ellas se tengan por conocidas - porque la cultura es producto de interacciones vivas y cambiantes, y porque la memoria humana suele fallar por muy bien intencionada que sea. También explicó que quizá muchas de las estudiantes del diplomado ya habían crecido en ámbitos urbanos y con títulos de profesión en campos de conocimiento occidental, y ello las tendría posiblemente alejadas y alienadas de su propia cultura; así que un ejercicio de registro de la cultura de sus ancestros desde un acercamiento más inquisitivo era primordial, como un plus en su proceso de reconexión con su identidad. A raíz de la buena experiencia de recurrir a la guía de mayores (y no a los antropólogos "expertos" en un pueblo indígena) se les pidió a las alumnas que estando en campo buscaran el consejo y acompañamiento de un sabio local que las asistiera en reconocer, grabar, registrar y contextualizar distintas 
formas de conocimiento indígena. Este ejercicio de acompañamiento resultó crucial en muchas investigaciones donde los sabios locales aparecieron como co-autores en los reportes finales de investigación.

Viendo en retrospectiva los debates, argumentos y desencuentros entre los diferentes actores del diplomado, caemos en cuenta que muchos de ellos están anclados, por un lado, en la polarizada relación con las ciencias sociales y la investigación social, y por otro, en la heterogénea condición de pertenencia al mundo indígena. La dicotómica diada adentro-afuera de la comunidad posicionaba de manera diferenciada a las alumnas en un universo fluido y poroso entre lo tradicional y lo moderno. Unas más "adentro" que otras (por ser comunitarias y rurales) y otras más "afuera” (por haber migrado a las ciudades a estudiar), pero todas al final pertenecientes a un colectivo fuertemente anclado en el proyecto político de mujeres indígenas del diplomado. Lo que ellas desarrollaron como su propia experiencia de apropiación ya es parte de lo que contextual y circunstancialmente pudieron lograr en la etnización de los métodos de investigación que emplearon.

\section{Reconstruyendo el gran Quillapi de la Memoria y las Mujeres Mapuches en la Recuperación de Tierras}

En esta sección queremos presentar las experiencias y pensamientos reflexivos de dos estudiantes que llevaron a cabo su proceso de investigación y adaptaron metodologías de maneras muy creativas. Las dos estudiantes tienen en común un interés en el papel de las mujeres en los procesos comunitarios de revitalización cultural, recuperación de tierras y autogobierno, e interés en la investigación social. Como facilitadoras de este Diplomado consideramos de gran relevancia traer a la luz las narrativas de las estudiantes sobre cómo se involucraron con sus comunidades, construyeron relaciones de confianza y produjeron obras finales de buena calidad. Ciertamente, arrojan luz sobre nuestro debate sobre la enseñanza de metodologías de investigación, ya que se posicionaron como investigadoras que abordaron el desafío de indagar desde dentro.

Mónica Michelena es una activista indígena charrúa de Uruguay. Es fundadora del Consejo de la Nación Charrúa (CONACHA), organización creada para dar voz a diferentes iniciativas comunitarias, con el fin de entablar un diálogo con el Estado uruguayo sobre el reconocimiento de la composición multiétnica del país. Uru- 
guay es un país sudamericano que se ha definido — hasta hace muy poco- como país "sin indios", debido al exterminio que el gobierno llevó a cabo a principios del siglo XIX, conocido como la "masacre de Salsipuedes". El movimiento indígena en Uruguay surgió al final de la dictadura militar en 1984, y su lucha más persistente ha sido la concienciación de la sociedad nacional uruguaya sobre su pasado indígena. En particular, la iniciativa de investigación de Michelena se centró en el papel que las mujeres - especialmente las ancianas - desempeñan en el proceso de restaurar las raíces de la identidad indígena charrúa. Los testimonios, las narraciones, los sueños y la reconstitución de rituales vinculados a la maternidad y a la crianza de los hijos son centrales en el proceso de recolección de piezas de un recuerdo que casi se desvaneció por la fuerza.

El título de su investigación fue "Charrúa Mujeres y Reconstrucción de la Memoria del Gran Quillapi”. Michelena introdujo una metáfora para referir el proceso colectivo de recolección de piezas dispersas de información en una narrativa más grande que abarca los insumos de varias familias miembros. La narrativa más grande es la memoria oral del pueblo charrúa, y el proceso de reconstrucción de la memoria oral se asemeja a la delicada costura de un tradicional "Charrúa Quillapi”. ¿Qué es un Quillapi? Una capa que estaba hecha de pequeños pedazos de cuero animal, cada uno impreso con símbolos de diferentes clanes familiares de guerreros. Aunque actualmente este tipo de atuendo ya no se elabora a mano, y no se utiliza de acuerdo con las tradiciones del pasado, se utiliza para ceremonias religiosas y está siendo resignificado por la nueva generación de charrúas. Según Michelena, "la reconstrucción de la memoria charrúa implica un cuidadoso proceso de coser fragmentos de memoria/ conocimiento. $Y$ en cada uno de nosotros hay fragmentos dispersos del todo" (entrevista, noviembre de 2015).

El pueblo charrúa en Uruguay es sobreviviente de una larga historia de genocidio y violencia. Los charrúas contemporáneos están deseosos de conocer y descubrir su propia historia y cosmovisión. En este proceso de reconstitución cultural, las mujeres de las comunidades rurales son titulares y guardianes culturales de prácticas locales que reflejan y tienen cierta continuidad con el pasado. Inspirado por este supuesto, se convirtió en crucial para el proyecto de investigación invitar a las mujeres rurales a involucrarse en un proceso dialógico de recuperación y re-narración sobre sus prácticas diarias, así como el aprendizaje de los mayores. Michelena considera que en la integración del "Gran Quillapi” que ella articuló hay una clara distinción entre las voces y miradas de mujeres charrúas urbanas y rurales. Mientras tanto, el primer grupo 
está tratando de encontrar maneras de volver a conectarse con su historia ancestral politizando su identidad en el mundo urbano; las mujeres rurales son portadoras de una tradición cultural que, aunque fragmentada, está presente como referente vivo del pueblo charrúa en un país que los niega.

A sugerencia de los facilitadores del Diplomado de colaborar con un experto local (tutor ancestral) para permitir el proceso de investigación en la comunidad, Michelena tomó la iniciativa de invitar a dos colaboradores en su proceso y cada uno desempeñó un papel diferente en la investigación de campo. Michelena se identifica como una mujer descendiente urbana charrúa que se trasladó a la ciudad para estudiar cuando tenía 18 años, y desde entonces ha seguido diferentes estrategias para volver a conectarse con sus raíces. Michelena fue la persona líder en el proceso de investigación y para ella dicho proceso fue un acto político. Como expresó en sus propias palabras "yo traje al grupo el punto de vista militante. Me sentía más como una persona limítrofe y fronteriza, mucho en relación a donde me coloco, a veces como una mujer charrúa, otras veces como un testigo externo, a veces como un actor activo interno" (entrevista, noviembre de 2015). La segunda persona fue Mary Correa, una líder local y miembro de la organización comunitaria. Ella asumió el papel de guía espiritual; era capaz de ver las cosas con una visión integral y unir piezas dispersas en una interpretación coherente. Mari —el tutor local - era un asesor espiritual y conocedor de la cultura charrúa. La tercera participante fue una joven estudiante doctoral — Andrea Olivera — destinada a apoyar y conducir su propia investigación sobre el proceso de revitalización cultural indígena. Olivera es antropóloga y su papel fue asegurar que los hallazgos estuvieran en diálogo con un marco teórico. Olivera procedió de una manera muy estructurada, ya que era la antropóloga profesional encargada del registro de datos en su bloc de notas.

La estrategia metodológica fue el resultado de varios diálogos entre Michelena, Olivera y Correa. Sobre la base de esos diálogos planearon cómo llevar a cabo la investigación de campo. El proceso de reconstitución cultural debe iniciarse a través de la sistematización de las prácticas culturales de las mujeres rurales, como la maternidad, la curación y el reconocimiento del territorio ancestral. Recogieron testimonios orales y realizaron entrevistas personales para recabar los recuerdos de los miembros de la familia. Se quedaron con las familias en sus casas durante dos o tres días. Además organizaron grupos focales y rituales de sanación por la noche. Todo el proceso tomó un mes.

En el sitio del genocidio de Salsipuedes localizaron pueblos en donde persisten descendientes charrúas. Michelena lo llamó "la ruta de supervivencia del pueblo 
charrúa”, ya que se trata de un territorio de desplazamiento y guerra. Las familias que viven allí describieron a Michelena sobre un conjunto de paseos por la tierra tradicional charrúa. Parece que los lugareños se organizaron en grupos para caminar por todo el territorio, con el fin de trazar sus recuerdos de la tierra. Las investigadoras invitaron a los pobladores a caminar a lo largo de los ríos y tierras de cultivo durante dos días. Esos paseos trajeron a los miembros de la comunidad sentimientos positivos y negativos por igual. Los miembros de la comunidad se dieron cuenta de la situación actual de su territorio ancestral que se divide en parcelas privadas y terratenientes. También reconocieron la deforestación y el estado actual de agotamiento de los recursos naturales.

El ejercicio de compartir los recuerdos familiares fue crucial para reunir diferentes relatos de la supervivencia charrúa. Después de la masacre de "Salsipuedes", los supervivientes transmitieron sus conocimientos de los padres a los niños. Los testimonios y los relatos variaban de comunidad a comunidad y entre familias. Michelena se dio cuenta de que cada madre y abuela tiene diferentes maneras de llevar a cabo rituales como la ofrenda del niño a la luna. Este ritual es muy importante para las familias charrúas e implica la ofrenda de un bebé recién nacido a la luna para conseguir la bendición y asegurare fuerza y buena salud. "Pedimos la bendición de la luna a nuestros hijos. Queremos asegurarnos de que nuestras prácticas se transmiten, aunque cada vez que se realiza, el ritual contiene menos información sobre el contexto, el propósito, la utilidad, la secuencia. En cierto modo, es algo alarmante" (entrevista, noviembre de 2015).

Una vez terminado el proceso de investigación, Michelena, Olivera y Correa se comprometieron en diversos esfuerzos para compartir los resultados con los miembros de la comunidad. Inicialmente, pensaron en invitar sólo a las mujeres de la comunidad, aunque éstas llegaron junto con sus parejas y familias. Sorprendentemente, los hombres querían tomar parte y estaban muy involucrados, porque hablaron profusamente de sus madres y de los recuerdos orales que heredaron de ellas. La participación de los miembros de la comunidad en la discusión sobre las prácticas culturales y la memoria tuvo un impacto profundo, porque desató la conciencia sobre aspectos importantes de su cultura que apenas discuten o intercambian colectivamente. "¿Sabes?, nunca hablamos de este tipo de cosas. Nos enfocamos principalmente en cómo organizarnos para reclamar nuestros derechos, pero nunca pensamos en la relevancia de discutir colectivamente sobre cultura y memoria. Ahora sabemos que esto es importante" (entrevista, noviembre de 2015). 
Michelena reflexiona cuidadosamente sobre lo que aprendió después del proceso de investigación. El proceso le ayudó a construir un fuerte vínculo con las otras dos participantes de la investigación. Las tres compartieron una experiencia que cambió sus vidas y creció el respeto por el papel que cada una jugó en la investigación. Correa reforzó sus vínculos con las familias de la comunidad y enriqueció su conocimiento sobre la cultura charrúa desde la perspectiva de las mujeres. Olivera se reconoció como una joven uruguaya en diáspora que tuvo la oportunidad de regresar a su tierra natal en busca de las raíces de sus antepasados. Michelena se hizo más consciente de su política como activista y líder; aprendió a apreciar el conocimiento de las mujeres de la comunidad que son humildes sobre lo que saben y, por lo mismo, guardan para sí información valiosa. Aprendió a ser respetuosa con el ritmo de la comunidad y el momento adecuado para solicitar información. Cuando visitó estas comunidades, Michelena recuerda que en ese momento estaba emergiendo como líder. La experiencia contribuyó a consolidar su liderazgo político en muchas formas, como el reconocimiento de los lugareños como una representante comprometida a dar visibilidad de la lucha charrúa en foros internacionales. Ahora Michelena considera que su misión principal es ayudar a los miembros de la comunidad a conducir sus propios procesos de investigación a través de métodos apropiados. La comunidad necesita recuperar sus historias y mitos, anotarlos en libros de texto y usarlos para enseñar a las generaciones más jóvenes de charrúas.

La siguiente investigación es la de Millaray Painemal Morales, de Chile. Milla es una joven líder feminista mapuche; se crió dentro de una familia de líderes mapuche muy reconocidos. Milla ha estado cultivando una trayectoria de liderazgo por su cuenta también en los movimientos campesinos y feministas. Estaba interesada en saber más sobre el papel de las mujeres en la recuperación de tierras de su comunidad a través de una serie de luchas y litigios al principio de 2000. El título de su trabajo final fue "Las luchas de las mujeres mapuches de Rucapangue para acceder a la tierra”. El pueblo mapuche es la nación indígena más importante de Chile $(1,500$ de 18 millones). Sus territorios tradicionales antes de la conquista española eran un área geográfica que comprendía 30 millones de hectáreas a lo largo de los dos lados de las montañas del extremo sur de los Andes. Las guerras coloniales, las dictaduras y la invasión de colonos han reducido sustancialmente sus tierras. Desde los noventa en Chile se produjo el retorno a un régimen democrático y se inició un lento proceso de restitución de tierras a las comunidades mapuche. La comunidad de Milla, Rucapangue, del municipio de Chol-Chol, al sur de Chile, inició un proceso de reclamación y 
restitución de su tierra tradicional que estaba en propiedad privada. Todo el proceso duró varios años, e implicó la ocupación de la tierra, enfrentamientos físicos y varias batallas legales.

Milla consideró cuidadosamente que era posible llevar a cabo este proyecto de investigación en la comunidad porque la gente local la conocía a ella y a su familia. Este elemento fue crucial porque los miembros de la comunidad están cansados y han crecido sospechando de los forasteros y los antropólogos. En este particular, Milla se ha convertido en defensora de la extracción del conocimiento colonial debido a su activismo, aunque se percibe como forastera debido a su educación urbana. Por lo tanto, Milla necesitó el apoyo de un consejero local/tutor local para presentarse a la comunidad y su nombre fue Héctor Maniqueo. Maniqueo resultó una pieza fundamental en el proceso de investigación porque participó activamente en la organización comunitaria para la recuperación de la tierra. Además, Maniqueo es hablante fluido del mapuche, lo que fue determinante para tener una conexión positiva con la comunidad y explicar el propósito de la investigación en el idioma local. Esta era una forma muy apropiada de conectarse con los locales y ganar su simpatía. Milla y Maniqueo, a través de la experiencia investigativa, construyeron un fuerte vínculo, ella compartió con él información legal importante para la comunidad y bibliografía; a cambio, él le mostró a Milla la manera de entrar en la comunidad de manera acertada y comprometida.

Según Milla, la colaboración con Maniqueo responde al tema de la dualidad y la complementariedad desde un punto de vista indígena, lo que significa que cada género tiene que cumplir su tarea asignada, y la voluntad colectiva prevalecerá mientras todos asuman su parte. Por un lado, la presencia de Maniqueo a lo largo del proceso fue beneficiosa porque era amable y conocía los caminos de la comunidad (cultura mapuche). Dirigió ceremonias espirituales y, estrictamente, instruyó a Milla sobre los protocolos de acogida en la comunidad. Por otro lado, Milla fue bienvenida porque la gente de la comunidad percibió su respeto, compromiso y voluntad de estar conectada. Los orígenes de Milla están allí, su madre es una campesina local y junto con la estudiante trabajan en una pequeña parcela que le fue asignada a la familia Paimenal.

Ellos fueron notificados sobre nuestra visita. La comunidad nos dio la bienvenida, y seguimos el protocolo proporcionando regalos que recibimos en la ciudad, como té mate. Les explicamos el propósito de nuestra visita, el propósito de la investigación. Querían conocer los resultados del diálogo y la negociación con el Estado 
para la asignación de tierras. La tarea crucial fue transmitir un mensaje positivo sobre la intención honesta de esta iniciativa. Más tarde nos invitaron a formar parte de un conjunto de talleres sobre la Convención 169 de la Organización Internacional del Trabajo (огт)". (Entrevista, noviembre de 2015)

En particular, Milla quería volver a visitar el tema de la participación de las mujeres mapuche en el proceso de reivindicación de tierras y dónde estaban después de la asignación de tierras en términos de organización colectiva. Ella quería analizar críticamente por qué una vez obtenida la tierra, a las mujeres se les desplaza de los roles de liderazgo, y vuelven a colocarlas en actividades productivas. La estudiante quería poner de relieve la racionalidad de las mujeres indígenas detrás de sus decisiones y acciones. Para conocer y al mismo tiempo reflexionar de manera colectiva, facilitó talleres y entrevistas en profundidad con mujeres y hombres agricultores. Para Milla, esta investigación no se refería solamente a las mujeres, sino a la naturaleza misma del vínculo entre hombres y mujeres en la comunidad. Ella tomó en serio el compromiso de trabajar juntos y en colaboración con ambos sexos, para desarrollar una imagen compleja del proceso de cultivo y cómo se relaciona con la política de reivindicación de tierras.

¿Cuáles son las lecciones de la experiencia de investigación? Según la estudiante, la lección más importante fue darse cuenta de cómo el conocimiento local es la principal fuente de información en cualquier proceso de investigación, mientras la teoría es sólo una pieza. Además, la construcción de una relación de confianza con los miembros de la comunidad debe estar en el centro del proceso de investigación. Milla tuvo el cuidado de iniciar respetuosamente la relación de colaboración con los lugareños y está segura de que durará, porque ha encontrado formas de incorporar a la población local de su comunidad a las actividades políticas de una organización mapuche a la que pertenece en la ciudad.

Además de las lecciones positivas, consideró que había mucho que mencionar, pero también que reflexionar sobre los sentimientos de incomodidad a los que trató de hacer frente debido a su posición liminal como líder indígena con una profesión y educación urbana. El elemento más destacado fue su renuencia a ser etiquetada, ni como intelectual ni como investigadora:

Yo sólo quiero ser un instrumento para resolver las necesidades de la comunidad. Soy una facilitadora no un investigadora, y me niego a que me pidan dar entrevistas porque yo no soy un informante local o una mujer indígena vulnerable que necesita ser salvada. Mi subjetividad me impulsa a rebelarme. No quiero ser 
Dolores Figueroa Romero y Araceli Burguete Cal y Mayor

famosa, mi único deseo es volver a mi comunidad. Me siento libre cuando vuelvo a casa de mi mamá y trabajamos en la parcela de la familia. (Entrevista, noviembre de 2015)

Esta posición la relacionaba con una crítica al diseño del diplomado. Ella sentía que el calendario de formación no contemplaba el tiempo para devolver a la comunidad los resultados de la investigación. Por eso, ella tuvo que encontrar otra ocasión para entregarles una copia impresa del informe que, aunque completo, no contenía las fotos de mujeres y de hombres que fueron entrevistados. Hablando en general, Milla también externó sentirse incómoda con la fase de escritura de la investigación, pues sentía presión para acabar y entregar una versión final. Toda esta presión evitó implicar a Maniqueo en discutir y organizar ideas para ser incluidas en la versión escrita. Milla se sintió constreñida también para cumplir con el requisito de la estructura del ensayo, pues a su parecer se trataba de un esquema estricto y Milla se preguntó si había otra manera de escribir y presentar información sensible para su comunidad. Milla sugirió manejar un CD con los testimonios de las personas como un formato alternativo para presentar los resultados de la investigación. ¿Cuál es el uso de una pieza escrita para la gente rural? Es crucial pensar en la comunicación oral y las técnicas de registro para socializar mejor los resultados y pensamientos reflexivos a nivel comunitario.

De las narrativas de Michelena y Milla quisiéramos rescatar los siguientes elementos. Ambas alumnas se sitúan de manera problemática en la liminaridad de la pertenencia, y siendo mujeres maduras de varios años de militancia, la crianza y vivencia urbana las ha llevado a transitar otros caminos. La investigación social para ellas ha sido una vía de retorno y de re-encuentro con su comunidad, su familia y los procesos políticos locales. La subjetividad de ambas — rebeldes - es compleja y se tensiona ante los imaginarios del poder que el conocimiento científico da al "experto social”, y que ciertamente ellas re-significan en relación a sus propios referentes político-vivenciales. Ambas reflexionan sobre los efectos posteriores de sus investigaciones y en los imaginarios que ellas generan en otros públicos. Para Michelena es evidente la necesidad de producir más y más investigaciones, pero de cara a fortalecer el proceso charrúa y apuntalar mejor la incidencia a nivel nacional por el reconocimiento cultural del pueblo charrúa por el gobierno uruguayo. Para Milla, su preocupación por el liderazgo de mujeres mapuches en su comunidad la lleva a ser vista como una experta en feminismo indígena, etiqueta que reniega y rechaza de manera enfática, siempre con la esperanza de crear su propio espacio identitario alejado de los estereotipos de 
las profesiones del conocimiento/poder. Ambas tuvieron la destreza de desarrollar procesos de investigación juiciosos, comprometidos y creativos que dan cuenta de lo importante que es generalizar la comprensión y enseñanza de métodos de investigación social entre actores y organizaciones sociales, y entre otras cosas, des-centralizar el oficio del antropólogo.

\section{Concluyendo analíticamente}

Esta sección la queremos dedicar a reflexionar sobre la etnización de los métodos de investigación social a la luz de los resultados de las investigaciones del diplomado y los elementos condicionantes que le dieron su forma final. Recapitularemos mencionando que, para nosotras, la etnización de métodos de investigación convencionales implica un proceso que va en dos sentidos: Primero, investigadoras no-indígenas que buscan influenciar enfoques, metodologías, herramientas y lenguaje de la investigación social convencional con el pensamiento de las sociedades indígenas; y segundo, que los aprendices indígenas adopten y aprendan métodos de investigación social a fin de realizar su propio levantamiento de datos con una visión desde adentro (Rappaport, 2015). Este ejercicio doble está inspirado por una visión crítica a la colonialidad de la investigación social que no se queda en la sola deconstrucción de la hegemonía occidental, sino que asume el compromiso político y estratégico de producir conocimiento culturalmente pertinente, novedoso, transformador e intercultural. Dentro del Diplomado, criticar la hegemonía de pensamientos occidentales - como el antropológico y el feministafue sólo el inicio de un proceso donde se buscó, desde el actuar colegiado de docentes, facilitadoras y alumnas, la re-semantización de procesos de investigación ligados al reconocimiento de saberes y experiencias propios, desde las múltiples matrices culturales que alimentan la indigeneidad latinoamericana.

Para la coordinación académica del CIESAS uno de los objetivos principales del diplomado fue que las estudiantes adquirieran habilidades para hacer investigación social de una manera culturalmente sensible, que fuera útil para sus luchas, sus pueblos y sus procesos; y ello requirió darle una especial centralidad a todo el ciclo que implicó la selección de temas, el proceso de investigación y la presentación de resultados al final del diplomado. El ejercicio de selección temática fue resultado de un largo proceso que inició con una carta de intención que las alumnas prepararon a fin de ser seleccionadas para cursar el diplomado. Posteriormente $-\mathrm{y}$ como resultado 
de los cursos presenciales y en línea- las alumnas pulieron y seleccionaron con más cuidado temas que conciliaron su curiosidad individual investigativa con el interés de la comunidad y organización, (compromiso colectivo) y que fueran realizables dentro de un tiempo prudente de recolección de datos. Este proceso no estuvo exento de conflictos y negociaciones, pues algunas alumnas no encontraban cómo armonizar su interés, su tiempo y sus recursos y el permiso de la comunidad con el requerimiento al cual debían responder. Las exigencias de cumplimiento y el calendario de trabajo del diplomado generaron ansiedad entre las alumnas por lo constreñido del tiempo y por lo estructurado del modelo para la entrega de resultados, que el Fondo Indígena requería para su presentación ante las instancias de financiamiento.

Otro elemento importante a mencionar es que las y los docentes y facilitadores del diplomado tuvieron a bien incentivar por distintos medios la reflexión colectiva e individual sobre la inter-seccionalidad de las identidades étnicas y de género, a fin de politizar los puntos de conexión entre la reproducción de la vida comunitaria indígena de sus pueblos, la relación con el mundo mestizo y las múltiples discriminaciones por ellas vividas en su etapa formativa y vida adulta. Es por ello que, a nuestro juicio, que el impacto de más largo aliento que el diplomado tuvo se ve reflejado en las estudiantes que, adoptando herramientas de investigación, se dieron a la tarea de explorar de manera crítica, propositiva y liberatoria las múltiples formas en que las mujeres indígenas se sitúan en el amplio escenario de la lucha de sus pueblos.

Las voces de Michelena y Milla son representativas de los dilemas éticos que la mayoría de las estudiantes pasaron. El proceso de investigación desarrollado por ellas no se limitó a la sola aplicación de una herramienta sino a la adopción de una nueva identidad, la del investigador que documenta y pulsa el poder del conocimiento producido desde adentro. En muchos sentidos, Michelena y Milla sintieron la responsabilidad de co-responder y de afanarse en dar un sentido promisorio y práctico con los resultados obtenidos. Igualmente la producción de un resultado final de investigación les dio la certeza de que habían adquirido la destreza para producir en el futuro más investigaciones, y desde el lente de las necesidades de su gente. Las dos alumnas después de este diplomado han tenido experiencias de investigación y trabajos colaborativos con un amplio espectro de aliados y de actores políticos, siempre reflexivas sobre las fronteras porosas del adentro y del afuera, y sobre todo, cómo construir vías para, si se está "afuera", volver a pertenecer al "adentro" de manera útil y transformativa. Acabe señalar que para un alto porcentaje del casi centenar de mujeres que cursó el diplomado y que no habían iniciado o concluido una carrera 
profesional, la experiencia de investigación del diplomado les motivó a fortalecer su formación académica.

La idea anterior hace eco del actuar organizado de las lideresas indígenas que reclaman un espacio autónomo y propio para discutir sobre sus necesidades de formación y lo que es política y discursivamente pertinente para su activismo. En esa búsqueda y experimentación con diversas formas de empoderamiento, las lideresas han buscado establecer diálogos y alianzas estratégicas con aliados, en este caso antropólogas y feministas no-indígenas, experimentando en colectivo la etnización de la investigación y el uso alternativo de categorías como complementaridad, feminismo y género. El diálogo al que hacemos referencia no se dio en el vacío, sino en el rechazo de formas de dominación históricas que median las relaciones entre investigadores mestizos y líderes indígenas, y también en contra de los privilegios explícitos que posicionan a las mujeres mestizas feministas como "las voceras" de los canales apropiados para la liberación de la mujer indígena (Hernández, 2007). Como participantes y facilitadoras de este diplomado, creemos que las alumnas/lideresas indígenas tuvieron el tiempo y el espacio para experimentar y visualizar horizontes de liberación tanto personales como colectivos.

\section{Lista de referencias}

Bertely, M. (2013). Debates conceptuales sobre educación multicultural e intercultural. En M. Bertely, G. Dietz \& M. Díaz (Eds.), Multiculturalismo y educación 2002-2011 (pp. 41-79). México: Asociación Nacional de Universidades e Instituciones de Educación Superior.

Brysk, A. (2000). From tribal village to global village: Indian Rights and International relation in Latin America. California: Stanford University Press.

Burguete, A. (2013). Miradas Críticas de Abya Yala (Vol. I y II). Bolivia: Universidad Indígena Intercultural/Agencia de Cooperación Alemana/Fondo para el Desarrollo de Pueblos Indígenas de América Latina y el Caribe.

Cervone, E. (2002). Engendering leadership: Indigenous Women leaders in the Ecuadorian Andes. En R. Montoya, L. J. Frazier \& J. Hurtig (Eds.), Gender's Place. Feminist Anthropologies of Latin America (pp. 179-196). Estados Unidos: Palgrave Macmillan.

Cervone, E., Garcés, A., Larrea, S., Morocho, A., Prieto, M., Shiguango, N... Yangol, 
D. (1998). Mujeres contracorriente. Voces de Líderes Indígenas. Ecuador: Centro de Planificación y Estudio Social.

Chilisa, B. (2002). Indigenous research methodologies. California: Sage.

Cumes, A. (2009). Multiculturalismo, género y feminismo: Mujeres diversas, luchas complejas. En A. Pequeño (Coord.), Participación y políticas de mujeres indígenas en América Latina (pp. 29- 52). Quito: Facultad Latinoamericana de Ciencias Sociales.

Cunningham, M. (Octubre, 2002). Mujeres Indígenas en el Derecho Internacional. Trabajo presentado en el Congreso Internacional de la Asociación de Estudios Latinoamericanos (LASA), Toronto.

Cunningham, M., Mendizábal, E., \& Martínez, G. (Coords.). (2015). Las líderes indígenas tejiendo realidades y fortaleciendo liderazgos 2013. México: Universidad Nacional Autónoma de México.

Dietz, G., \& Martí i Puig, S. (2013). Empoderamiento y Educación Superior en contextos interculturales en México. Barcelona: Bellatierra.

Dietz, G., \& Mateos, L. (2015). Entre culturas, entre saberes, entre poderes: la etnografía reflexiva en el acompañamiento de procesos de interculturalidad educativa. En X. Leyva, J. Alonso, A. Hernández, A. Escobar, A. Köhler, A. Cumes... W. Mignolo (Eds.), Prácticas otras de conocimiento(s). Entre crisis y guerras (Tomo III, pp. 281-313). Chiapas, México: Retos.

Dixon, B., \& Sánchez, N. (2003). Género desde la visión de las mujeres indígenas. Nicaragua: Centro de la Mujer Indígena Multi-étnica/Universidad de las Regiones Autónomas de las Costa Caribe de Nicaragua.

Figueroa, D. (2006). Interview with Mirna Cunningham Kain. International Feminist Journal of Politics, 8(4), 618-626.

Figueroa, D. (2010). Propuesta para el Postgrado: "Fortalecimiento del Liderazgo de las Mujeres Indígenas". Bolivia: Fondo Indígena/Universidad Indígena Intercultural/Agencia de Cooperación Alemana GIz.

Guidugli, S., \& Cunningham, R. (2009). Propuesta Metodológica para Desarrollar Procesos de Aprendizaje en el Trabajo con Pueblos Indígenas. Bolivia: Fondo Indígena/ Universidad Indígena Intercultural/Agencia de Cooperación Alemana GIZ.

Hale, C. (2008). Reflexiones hacia la práctica de una investigación descolonizada. En Centro de Estudios Superiores de México y Centroamérica-Universidad de Ciencias y Artes de Chiapas (Ed.), Anuario 2007 (pp. 299-315). México: Editor. Recuperado de https://goo.gl/J23 Fcr 
Hernández, A. (Noviembre, 2007). Socially Committed Anthropology from a Dialogical Feminist Perspective. Trabajo presentado en el panel "Critically Engaged Collaborative Research: Re-making Anthropological Practice” de la Reunión Anual de la Asociación Americana de Antropología, San José, California.

Jimeno, M., Murillo, L., \& Martínez, M. (Eds.). (2012). Etnografías contemporáneas. Trabajo de Campo. Bogotá: Centro de Estudios Sociales-Facultad de Ciencias Sociales-Universidad Nacional de Colombia.

Lugones, M. (2015). Hacia metodologías de la decolonialidad. En X. Leyva , J. Alonso, A. Hernández, A. Escobar, A. Köhler, A. Cumes... W. Mignolo (Eds.), Prácticas otras de conocimiento(s). Entre crisis y guerras (Tomo III, pp. 75-92). Guadalajara, México: Retos.

Marchand, M., \& Parpart, J. (1999). Feminism, Postmodernism, and Development. Reino Unido: Routledge.

Martí i Puig, S. (2004). Sobre la emergencia y el impacto de los movimientos indígenas en las arenas políticas de América Latina. Algunas claves interpretativas desde lo local y lo global. En J. M. Sanahuja \& S. Martí i Puig (Coords.), Etnicidad, autonomía y gobernabilidad en América Latina (pp. 167-196). España: Universidad de Salamanca.

Mato, D. (2013). Diversidad Cultural, Interculturalidad y Educación Superior en América Latina. Contextos y Modalidades en la Colaboración Intercultural, Logros y Desafíos. En S. Hernández, M. I. Ramírez, Y. Manjarrez \& A. Flores (Coords.), Educación intercultural a nivel superior: Reflexiones desde diversas realidades latinoamericanas (pp.155-176). Puebla, México: Universidad del Estado de Puebla.

Medina, P., \& Baronnet, B. (2013). Movimientos decoloniales en América Latina: un balance necesario desde las pedagogías interculturales emergentes en México. En M. Bertely, G. Dietz \& M. Díaz (Eds.), Multiculturalismo y educación 20022011 (pp. 415-448). México: Consejo Mexicano de Investigación Educativa/ Asociación Nacional de Universidades e Instituciones de Educación Superior.

Niezen, R. (2009). The Rediscovered Self. Indigenous Identity and Cultural Justice. Montreal: McGill-Queen's University Press.

Pallares, A. (2007). Contesting Membership: Citizenship, Pluriculturalism (s), and the Contemporary Indigenous Movement. En A. K. Clark \& M. Becker (Eds.), Highland Indians and the State in Modern Ecuador (pp. 139-154). Pittsburgh: University of Pittsburgh Press. 
Rappaport, J. (2015). Más allá de la observación participante: la etnografía colaborativa como innovación teórica. En X. Leyva, J. Alonso, A. Hernández, A. Escobar, A. Köhler, A. Cumes... W. Mignolo (Eds.), Prácticas otras de conocimiento(s). Entre crisis y guerras (Tomo III pp. 323-352). Chiapas, México: Retos.

Segato, R. (2014). Género y colonialidad: del patriarcado de bajo impacto al patriarcado moderno. En M. Belausteguigoitia \& M. J. Saldaña (Coords.), Desposesión: género, territorio y luchas por la autonomía (pp. 321-361). México: Universidad Nacional Autónoma de México.

Sikkink, K. (1993). Human Rights, Principled Issue-Networks, and Sovereignty in Latin America. International Organization, 47(3), 411-441.

Smith, L. T. (1999). Decolonizing Methodologies. Research and Indigenous People. Reino Unido: Zed Books.

Valladares, L. R. (2008). Los derechos humanos de las mujeres indígenas. De la aldea local a los foros internacionales. Alteridades, 18(35), 47-65.

Varese, S., Delgado, G., \& Meyer, R. L. (2008). Indigenous Anthropologies beyond Barbados. En D. Poole (Ed.), A Companion to Latin American Anthropology (pp. 375-399). Oxford: Blackwell Publishing.

Vargas, L. V., López, M., \& Burguete, A. (2012). Experiencia de formación de liderazgo de mujeres indígenas en México y América Latina. Mujeres articuladas y alianzas con resultados. México: Alianza de Mujeres Indígenas de Centroamérica y México/Foro Internacional de Mujeres Indígenas/Cátedra Indígena Itinerante/ Centro de Investigaciones y Estudios Superiores en Antropología Social.

Walsh, C. (2009). Interculturalidad, Estado, Sociedad: Luchas (de)coloniales de nuestra época. Quito: Universidad Andina Simón Bolívar/Abya-Yala.

Walsh, C., Mignolo, W., \& García, A. (2006). Interculturalidad, descolonización del estado y del conocimiento. Buenos Aires: Signo. 